\title{
LA FUNDACIÓN ROCKEFELLER Y EL ORGANISMO DE SALUD DE LA SOCIEDAD DE NACIONES: ALGUNAS CONEXIONES ESPAÑOLAS (*)
}

\author{
Paul Weinding. Welcome Unit for the History of Medicine. Oxford \\ (*) El presente trabajo es fundamentalmente una versión adaptada del artículo titulado Philanthropy and World Health: the \\ Rockefeller Foundation and the League of Nations Health Organisation. Minerva; vol. 35 (edición especial publicada \\ por el D. Stapleton sobre filantropía y desarrollo de instituciones) (en prensa).
}

Tras la Primera Guerra Mundial, fueron pocos los países que no se beneficiaron de las subvenciones de la Fundación Rockefeller (FR), una de las instituciones más activas a la hora de internacionalizar la sanidad pública. En España, entre 1917 y 1950, este hecho queda demostrado por la concesión de 19 becas destinadas a administradores de la sanidad pública, así como de diez becas adicionales destinadas a trabajadores de los laboratorios de sanidad pública, a técnicos sanitarios y a responsables de la elaboración de estadísticas médicas. Además de conceder subvenciones a países específicos, la Fundación Rockefeller aportaba más de la tercera parte del presupuesto del Organismo de Salud de la Sociedad de Naciones (League of Nations Health Organisation - LNHO), y sus becas internacionales sirvieron para desarrollar un grupo de expertos de carácter internacional en materia de política sanitaria. Existía una relación compleja entre los objetivos cducativos de la Fundación, su apoyo a la investigación científica y su función de exportación de los programas de sanidad pública de Estados Unidos ${ }^{1}$.

La Fundación Rockefeller contaba con una serie de áreas prioritarias: Estados Unidos, China, Europa del Este y Latinoamérica. La sanidad pública española recibía financiación de la FR; el alcance de los proyectos emprendidos todavía es objeto de investigaciones por parte de los historiadores españoles. El objetivo del presente trabajo es el de plantear deter- minados problemas generales para, posteriormente, evaluar algunas de sus repercusiones en relación con el caso español. Analizaré las estructuras características en materia de sanidad pública del período de entreguerras y cómo se produjo el hecho de que cl nuevo Organismo de Salud de la Sociedad de Naciones fuera financiado principalmente a través de una institución privada de carácter filantrópico. Los fondos aportados por la Fundación Rockefeller sirvieron para mejorar la posición de los expertos de salud pública a la hora de modernizar la sanidad pública a nivel global. Cada vez hay más literatura sobre la función de la FR en el desarrollo de las instituciones de sanidad pública. La Commission Américaine pour Combattre la Tuberculose tuvo una influencia muy relevante sobre la sanidad pública francesa ${ }^{2}$. Asimismo, reseñaré unos pocos ejemplos extraídos del caso español. Por último, examinaré algunos problemas de tipo administrativo, profesional y político.

La provisión de unos servicios sanitarios y de bienestar de carácter igualitario estaba encaminada a reducir los conflictos sociales internos y las tensiones internacionales. No se trataba sólo de contener la propagación de las infecciones: era necesario desarrollar unos acuerdos sociales positivos en materia de salud en los nuevos estados europeos del bienestar. Los Ministerios de Sanidad debian garantizar la cohesión social y promover «la salud en el más amplio sentido de la palabra». En toda 
Europa se impuso un modelo de administración centralizada de la salud por parte del estado, lo que originó la fundación de nuevos ministerios de sanidad. En Europa Central, como legado del Imperio Austro-Húngaro, dichas instituciones ya servían para coordinar la producción de vacunas así como para tomar otras medidas de carácter científico y de atención primaria. Los expertos médico-militares de los Aliados procedentes de Estados Unidos, Gran Bretaña, Francia, Italia y Japón se reunieron en Cannes en abril de 1919, con el fin de crear un Consejo Internacional y una Oficina de Salud Pública; lo que muestra la naturaleza altamente politizada de dichos programas. En su calidad de país no beligerante durante la Primera Guerra Mundial, España no tenía el estatus ni de víctima ni de país vencido, por lo que no se vio beneficiada por los programas de ayuda de emergencia. España había actuado de intermediaria entre las Potencias Centrales y los Aliados durante la guerra en relación con cuestiones tales como las condiciones sanitarias de los prisioneros de guerra. Los diplomáticos españoles reivindicaron sus aspiraciones a ocupar un sitio permanente en el Consejo de la Sociedad de Naciones en 1925, en respuesta a la admisión de Alemania, amenazando con abandonar el organismo si sus reivindicaciones se veían rechazadas. No obstante, para participar en el Organismo de Salud (League of Nations Health Organisaton - LNHO) no era necesario ser miembro de la Sociedad de Naciones.

¿Cómo se produjo la participación de la FR en la Sociedad de Naciones? Después de todo, Estados Unidos mantuvo una política aislacionista durante el período posterior a la guerra $\mathrm{y}$, además, Estados Unidos no era miembro de la Sociedad de Naciones. Existen determinadas funciones sanitarias que tienen un carácter internacional; los gobiernos nacionales no pueden llevarlas a cabo; dichas funciones resultan esenciales para la salud de las personas de todos los lugares. Estos sentimientos fueron manifestados por Wickliffe Rose a Frederick Russell, del Consejo Internacional de Salud (International Health Board - IHB) de la Fundación Rockefeller en 1922. Frente a la miseria y a las epidemias masivas existentes tras la Primera Guerra
Mundial, la FR consideraba la salud internacional como un ámbito que requería un nuevo tipo de organización. Las organizaciones de carácter filantrópico resultaban ideales a la hora de realizar tareas más allá de las fronteras de los estados. El problema consistía en si dicho ámbito internacional hacía referencia únicamente a una serie limitada de funciones que se encontraban más allá de la capacidad de los estados-nación - subsidiariedad en el lenguaje moderno europeo-, o, por el contrario, un organismo internacional de salud podía establecer unos modelos óptimos en materia de provisión de servicios de sanidad pública por encima de las autoridades estatales, a menudo negligentes o deseosas de limitar el gasto. De modo que se empezaron a desarrollar unas relaciones muy innovadoras entre la Fundación Rockefeller y el Organismo de Salud de la Sociedad de Naciones, que sirvieron para configurar los sistemas de salud pública de muchos países. Aunque el Consejo Internacional de Salud trató de mejorar los servicios sanitarios, sus emprendedores directivos se dieron cuenta de que, para conseguir cualquier mejora de los sistemas nacionales de sanidad, era necesario desarrollar unos modclos internacionales: con el fin de lograr progresos en las ciencias médicas y en epidemiología, era necesario desarrollar modelos internacionales, realizar estudios en colaboración y coordinar la asistencia médica internacional. Se desarrolló una relación especial entre la Fundación Rockefeller y el Organismo de Salud de la Sociedad de Naciones (League of Nations Health Organisation - LNHO), en la medida en que la FR consideraba a éste último como un medio para progresar en su estrategia de aumentar los niveles globales de salud en todo el mundo, mediante la mejora de los conocimientos científicos y médicos y de los recursos institucionales en materia de grupos selectos de expertos. Al contar con una visión más amplia de la cooperación internacional entre los expertos, los responsables de la FR consideraron inadecuados el legado de tratados intergubernamentales y de acuerdos sanitarios heredados del período anterior a la Primera Guerra Mundial. Dichos responsables opinaban que era necesario constituir una organización permanente que fuera capaz de actuar en relación con los nuevos descubri- 
mientos científicos y con la divulgación de los principios organizativos de la sanidad pública. El citado objetivo referido a la creación de una institución de este tipo explica los motivos por los que la Fundación Rockefeller, con su universalismo filantrópico, resultó crucial a la hora de proporcionar asistencia al Organismo de Salud de la Sociedad de Naciones ${ }^{5}$.

Una cuestión clave era si la sanidad internacional podría dejarse en manos únicamente de las instituciones filantrópicas, como la nueva Cruz Roja Internacional, o, por cl contrario, la nueva secretaría de salud debía ser responsable ante la Sociedad de Naciones sólo en su calidad de representante de los estados miembros. Y, en este último caso, qué ocurriría con las principales potencias mundiales, como Estados Unidos, Alemania y la Unión Soviética, que no eran miembros de la Sociedad de Naciones. Y qué ocurriría con los territorios coloniales y bajo mandato británico y francés que tenían una influencia dominante en la Sociedad de Naciones. Debido a la tendencia a consolidar los sistemas nacionales de bienestar bajo la autoridad de los ministerios de sanidad, un organismo internacional de salud podía convertirse en un intruso no deseado. Existía el peligro inherente de que los funcionarios gubernamentales, con una perspectiva minimalista, fueran incapaces de compartir dicha visión ampliada de los expertos en materia de sanidad pública internacional.

\section{DESARROLLO DE LA ORGANIZACIÓN}

Debido a la devastación que se produjo en Europa como consecuencia de la Primera Guerra Mundial, los responsables de la FR argumentaban que mientras que la sanidad pública de Estados Unidos se había desarrollado rápidamente, la organización europea de la sanidad pública estaba teñida de un imperialismo anticuado. Los responsables criticaban la estrechez de la perspectiva médica de la microbiología de Pasteur y de Koch. La Fundación Rockefeller no retrocedió ante el incremento del aislacionismo de Estados Unidos a principios de la década de 1920, pero no consideró adecuado asumir un papel demasiado prominente. La
Fundación Rockefeller era prácticamente una organización gestionada y formada por personal estadounidense y, a pesar de su perspectiva dinámica a nivel internacional, se veía limitada por factores domésticos derivados de la situación de Estados Unidos. No obstante, los responsables sobre el terreno de la FR vinculaban el desarrollo de las ciencias médicas a las actuaciones internacionales. Los responsables de la Fundación Rockefeller consideraban que: «Todo depende de la fortaleza permanente de los gobiernos democráticos.»

Ludwick Rajchman, el carismático Director del nuevo Instituto de Higiene de Varsovia, presidió la Comisión para Epidemias de la Sociedad de Naciones desde 1921, desarrollando con habilidad un Organismo de Salud de la Sociedad de Naciones de carácter permanente a partir de una institución temporal de emergencia. Ludwick Rajchman se distanció de sus patrocinadores franco-británicos e intensificó las relaciones con la Fundación Rockefeller ${ }^{7}$. Asimismo, trabajó en colaboración con los responsables de la FR, Rose y Gunn, con el fin de establecer una estrategia común para construir institutos centrales de salud pública, así como una estructura coordinada y dividida en diversos niveles de clínicas y hospitales públicos. El Organismo de Salud de la Sociedad de Naciones combinó las funciones de investigación y de organización, lo que supuso que tanto el departamento de ciencias médicas de la Fundación Rockefeller como el Consejo Internacional de Salud pudieran financiar, aproximadamente a partes iguales desde 1922 hasta 1934, al Organismo de Salud de la Sociedad de Naciones, contribuyendo cada uno de ellos con más de un millón de dólares USA durante esta etapa de construcción.

La alternativa de la Fundación Rockefeller a establecer un telón de acero sanitario de puntos de desinfección y de cuarentena era la de proporcionar asistencia a un programa de salud positiva basado en la medicina preventiva. Europa del Este se encontraba preparada para aplicar el nuevo régimen de sanidad pública diseñado en el Escuela de Salud Pública de la Universidad John Hopkins ${ }^{8}$. Los sistemas de formación en las líneas americanas debían servir para sustituir la dependencia de las ins- 
talaciones de salud pública y de la educación médica existentes en Alemania y Austria que había prevalecido hasta 1914. Los objetivos del Consejo Internacional de Salud fueron proporcionar asistencia «a los organismos oficiales de sanidad pública para que desarrollen medidas administrativas» y realizar investigaciones de campo encaminadas a la prevención de determinadas enfermedades, como la anquilostomiasis, la fiebre amarilla y la malaria. Aunque el Consejo se dio cuenta de que era necesario adaptarse a «las condiciones, las tradiciones, las necesidades y las costumbres locales», se dedicó fundamentalmente a divulgar los métodos estadounidenses en materia de sanidad pública, estrategia que los responsables de la Fundación Rockefeller consideraban que iba más allá de la perspectiva limitada de la bacteriología alemana o de la microbiología francesa. Por lo tanto, la FR era proclive a sustituir otras influencias extranjeras relevantes en España como la microbiología francesa. Los institutos de higiene debían ser los baluartes contra la propagación de epidemias al actuar como núcleos de una nueva infraestructura de clínicas y de centros de salud compuestos por médicos y personal de enfermería formados en los modernos métodos de salud pública. La mejora de la nutrición y de la higiene personal debían promover la resistencia física del cuerpo ante las infecciones, mientras que el amplio programa social y de medicina preventiva estaba destinado a reforzar la estabilidad política mediante la construcción de unas estructuras administrativas internas.

El equilibrio de poder entre los expertos y los representantes de los estados quedó sin resolver. No se zanjó el debate sobre si el organismo internacional de salud debía ser únicamente una cámara dc compensación en relación con la información generada por los nuevos ministerios de sanidad o, por el contrario, debía establecer unos modelos y unas directrices óptimas, así como actuar como el organismo impulsor de la reforma sanitaria internacional. Rajchman argumentó que el Organismo de Salud de la Sociedad de Naciones debía realizar tareas de carácter más esencial. En la medida en que la Fundación Rockefeller se encontraba cercana a sus posturas referidas al desarrollo de un conjunto de expertos internacionales en materia de salud pública, la financiación no resultó problemática. El entusiasmo de los principales directivos del Consejo Internacional de Salud, en particular, Wickliffe Rose y Selskar Gunn, permitieron a Rajchman sobrepasar las limitaciones de las políticas más minimalistas de los británicos y de los franceses en relación con el Organismo de Salud de la Sociedad de Naciones. No obstante, una vez que se constató que los importantes fondos asignados generaban un choque entre la autonomía de los expertos y la responsabilidad política, la situación se convirtió en problemática ${ }^{9}$.

El Comité de Salud de la Sociedad de Naciones estaba presidido por el experto danés en serología Madsen, e incluía distinguidos científicos médicos, como el experto alemán en malaria, Bernhard Nocht, y Carlos Chagas del Instituto Osvaldo Cruz. En 1923, España estaba representada en el Comité de Salud por Angel Pulido, antiguo Director General del Departamento de Sanidad Pública de Madrid. En 1924, éste se vio sustituido por Gustavo Pittaluga de la Facultad de Medicina, quién asistió a casi todas las reuniones del Comité de Salud hasta $1936^{10}$. Pittaluga jugó un papel activo en la comisión para la malaria y promovió la extensión de los trabajos del Organismo de Salud de la Sociedad de Naciones a Cuba y a África" ${ }^{11}$.

\section{PREVENCIÓN DE LAS EPIDEMIAS}

Los funcionarios médicos de los ministerios de Francia y de Gran Bretaña centraron sus expectativas de crear una organización eficaz en materia de sanidad internacional en la Oficina Internacional de Salud Pública con sede en París, fundada en 1907, para regular los acuerdos internacionales sobre cuarentenas. En 1924, España y Portugal se encontraban entre los cuarenta miembros de esta organización. Los británicos y los franceses admitían que la Sociedad de Naciones podía aumentar sus competencias al respecto mediante una comisión para epidemias, con el fin de hacer frente a la amenaza del tifus y del cólera procedente de Rusia, lo que se corres- 
pondía con su política de consolidación del nuevo estado polaco como un estado tapón frente al imperialismo alemán y al comunismo soviético. El Organismo de Salud de la Sociedad de Naciones fue creado en junio de 1921. Rose, que se reunió con Rajchman en marzo de 1922, favoreció que el programa de la Fundación Rockefeller para Europa del Este fuera puesto bajo la autoridad de la nueva Oficina de Salud de la Sociedad de Naciones ${ }^{12}$. Rose respaldó un elaborado programa de intercambios del personal de la sanidad pública y los acuerdos sobre información en materia de epidemias ${ }^{13}$.

En junio de 1922, la Fundación Rockefeller destinó fondos al Organismo de Salud de la Sociedad de Naciones con el fin de proporcionar asistencia a un servicio de información epidemiológica y de elaboración de estadísticas en materia de salud pública. La siguiente etapa consistió en el compromiso de financiar los intercambios internacionales de personal de la sanidad pública ${ }^{14}$. Las becas de la Fundación Rockefeller contribuyeron a estabilizar el todavía provisional Organismo de Salud de la Sociedad de Naciones, cuyo presupuesto inicialmente se establecía con carácter anual ${ }^{15}$. En el momento de la retirada de Rose del Consejo Internacional de Salud, en 1923, empezó una nueva etapa con Frederick Russell. Mientras que Rose era por sus orígenes un filósofo y un educador, Russell había sido un funcionario médico militar con formación en materia de investigación de laboratorio. Por fortuna, dicho cambio coincidió con el cada vez mayor énfasis técnico de Rajchman en los estudios de normalización basados en la investigación de laboratorio. Selskar Gunn, que continuaba siendo responsable del programa europeo, admiraba la calidad visionaria y radical de los programas de Rajchman ${ }^{14}$.

Gunn aprobó los contactos con reformadores radicales en materia de salud, tales como el carismático yugoslavo, Andreas Stampar, un socialista de tendencias radicales. Stampar pretendía trasponer las unidades sanitarias de condado y las innovaciones sanitarias de Estados Unidos, tales como los «Kentucky closets» (Gabinetes Médicos de Kentucky), a la nueva Yugoslavia. La visión de Stampar de la atención primaria de salud impartida por las instituciones públicas era compartida por la Fundación, que también tenía una opinión crítica de la práctica médica privada $\mathrm{y}$, en su lugar, confiaba en un sistema de sanidad pública con una base científica ${ }^{17}$. Los médicos debían ser «trabajadores sociales» en vez de estar confinados improductivamente en hospitales y clínicas. Aunque Stampar creía en una administración nacional unitaria, la tendencia hacia la organización federal fue reafirmada por una propuesta de programa para la creación de un nuevo centro de formación de salud pública, un instituto epidemiológico y una oficina central de sanidad para Croacia y Eslovenia, con sede en Zagreb y bajo la presidencia de Stampar. ${ }^{18}$

El programa de Zagreb formaba parte de un programa internacional del Consejo Internacional de Salud destinado a proporcionar asistencia a los institutos centrales de higiene del estado en su calidad de centros de investigación, de administradores de la sanidad pública y de proveedores de atención primaria de salud. Se crearon nuevos institutos en Varsovia, Praga y Budapest. No resulta sorprendente observar la colaboración del Organismo de Salud de la Sociedad de Naciones y de la Fundación Rockefeller a la hora de patrocinar dichos programas. La Escuela de Higiene de Madrid se vio influenciada por desarrollos internacionales más amplios. En 1933, Carl Prausnitz (que emigró a Gran Bretaña cuando se convirtió en médico de familia) publicó su informe para la Sociedad de Naciones: «La enseñanza de la medicina preventiva en Europa» (The Teaching of Preventive Medicine in Europe). Prausnitz estableció la función del Instituto de Higiene de Madrid a la hora de formar a los responsables médicos, así como a los inspectores de sanidad, a las comadronas y al personal de enfermería de los hospitales. Asimismo consideraba los cursos impartidos en Madrid como bastante similares a los desarrollados en otros países ${ }^{19}$.

A principios de la década de 1930, la Fundación Rockefeller deseaba distanciarse del Organismo de Salud de la Sociedad de Naciones que adoptó una postura cada vez más radical. La FR argumentó que el Organismo de 
Salud de la Sociedad de Naciones no constituía un caso especial sino que era, fundamentalmente, un ministerio de sanidad pública similar al de cualquier otro gobierno. Cualquier donación de la Fundación Rockefeller al Organismo de Salud de la Sociedad de Naciones otorgaría demasiada autonomía a este organismo: por lo tanto, todas las ayudas debían tener un carácter temporal y la FR empezó a estudiar estrategias de ruptura ${ }^{21}$. Un indicador de este hecho es que, en 1931, Russell solicitó que el Consejo Internacional de Salud ya no apareciera reseñado en el Anuario Internacional de Salud (International Health Yearbook), que se dedicó a realizar estudios sobre los servicios nacionales de sanidad ${ }^{21}$. La opinión de Rajchman, en el sentido de que la Sociedad de Naciones no debía ser considerada meramente como cualquier otra institución gubernamental: sus responsabilidades específicas «en relación con el bienestar de la humanidad y con la prevención de las guerras» la convertían en un caso único a la hora de recibir la financiación de la Fundación Rockefeller ${ }^{22}$.

\section{LA CONSTRUCCIÓN DE UNA «COMUNIDAD EPISTÉMICA»}

En 1923 se redactó un acuerdo con el fin de introducir y de proporcionar formación a técnicos de estadística médica, en la medida en que existía una necesidad de alcanzar una uniformidad y una capacidad de comparación a nivel internacional. La Fundación Rockefeller financió intercambios periódicos para que técnicos de estadística médica estudiasen en Suiza, en Francia y en Holanda; y los problemas referidos a los datos estadísticos sobre tuberculosis y sobre nacimientos de mortinatos eran considerados prioritarios ${ }^{23}$. Aunque el programa destinado a crear una Oficina de la Sociedad de Naciones, formada por un experto alemán en medicina tropical en Moscú, no llegó a fructificar, en 1925 se alcanzó un acuerdo para que la FR proporcionase financiación a la Oficina Oriental en Singapur. La Fundación Rockefeller proporcionaba asistencia al Organismo de Salud para la elaboración de los informes epidemiológicos semanales, mensuales y anuales. Los informes mensuales del Organismo de Salud incluían una sección sobre las tasas generales de mortalidad, la incidencia de la peste, el cólera, la fiebre amarilla, la viruela y la sífilis, la mortalidad infantil, así como los indicadores de la salud de otros grupos de edad tales como los fallecimientos debidos a fiebre puerperal, y las tendencias de la mortalidad en las grandes ciudades, con inclusión de las enfermedades de los bebés y de los niños. En 1937, el establecimiento de la Oficina Oriental implicó que el $72 \%$ de la población mundial estuviera cubierto por las estadísticas médicas del Organismo de Salud de la Sociedad de Naciones. El Anuario, publicado a partir de 1925 , proporcionaba una útil panorámica de la sanidad pública nacional y de los servicios sanitarios profesionales. Cada publicación contenía un capítulo sobre la sanidad pública española, que abarcaba el índice de natalidad, la tasa de mortalidad y la tasa de mortalidad infantil, y el presupuesto ${ }^{24}$.

El programa de modelos biológicos compensó la falta de laboratorios existente en Ginebra. Los laboratorios del Instituto Nacional de Investigación Médica de Londres y el Instituto Estatal de Serologia de Copenhague, eran los principales responsables del establecimiento de unos modelos biológicos. Las pruebas normalizadas de la sífilis no sólo se desarrollaron en los centros europeos como el Instituto Pasteur, sino también en lugares tan lejanos como Montevideo. Durante la década de 1920, el Organismo de Salud se centró en los modelos biológicos relativos a medicamentos, como la insulina y el salvarsán, las vacunas y los preparados hormonales. Las enfermedades como la sífilis fueron objeto de atención desde el punto de vista del desarrollo de unos procedimientos normalizados para la realización de las pruebas, tanto para el diagnóstico Wassermann y las pruebas de floculación de la sífilis como para unos regímenes normalizados de tratamiento como, por ejemplo, para el salvarsán. La sífilis, con unas formas y una sensibilidad humana de carácter muy variable ante la espiroqueta causal, ilustra bien los defectos de una perspectiva demasiado centrada en los países europeos: en África se producía una carencia de instalaciones y no se podía confiar en que los pacientes siguieran cursos prolongados de tratamiento con medi- 
camentos que no estaban disponibles de forma regular ${ }^{25}$.

El desarrollo de vacunas y el trabajo inmunológico se continuó llevando a cabo en la década de 1930. Zinsser y Nicolle crearon un eje Francia-Polonia-Estados Unidos para desarrollar la colaboración internacional en materia de investigación sobre el tifus, con ramificaciones en España, Chile, Bolivia, Méjico y China. Zinsser tenía la intención de probar su vacuna en España a lo largo de 1937, bajo los auspicios del Organismo de Salud de la Sociedad de Naciones, habiendo obtenido el apoyo del Ministerio de Sanidad de la República Española. Otros lugares en los que se realizaron ensayos fueron Argelia y Marruecos, Rumania, Turquía y China. Aunque Zinsser abandonó el proyecto de España, luchó por establecer un laboratorio de campo en Europa, con el fin de probar su nueva vacuna y dc proporcionar formación a jóvenes investigadores procedentes de Rumania, Francia y Polonia, bajo los auspicios de la Sociedad de Naciones ${ }^{20}$. Una vez desechada esta posibilidad, Zinsscr tenía la esperanza de que el Instituto Pasteur se convirtiera en el centro de producción de vacunas para Oriente Medio y para Europa del Este ${ }^{27}$.

Con la crisis económica, el programa de normalización se amplió hasta incluir estudios sobre las carencias sociales, la dieta, las condiciones globales de salud de las poblaciones y los factores que afectan a la incidencia de las enfermedades. El Organismo de Salud de la Sociedad de Naciones pretendía establecer los requisitos dietéticos mínimos, así como los modelos óptimos para los diferentes grupos de edad y las diversas profesiones. Las circunstancias de la Depresión demostraron el potencial social de los modelos internacionales. Los bioquímicos eran los responsables de calcular los modelos de nutrición, y sus estudios sirvieron para establecer los factores alimentarios individuales necesarios para una dieta saludable y los requisitos en materia de cantidad. Las conferencias sobre los modelos vitamínicos, celebradas en Londres en junio de 1931 y en junio de 1934, establecieron unas unidades tipo para las vitaminas $\mathrm{A}, \mathrm{B} 1, \mathrm{C}$ y $\mathrm{D}^{28}$. El Comité para la Tuberculosis del Organismo de
Salud muestra un movimiento del centro de interés desde las cuestiones técnicas preponderantes en la década de 1920 hasta las cuestiones sociales en la década de 1930. El Comité evaluó la eficacia de la vacunación BCG realizada en Francia, que analizó a la luz de los datos estadísticos proporcionados por el Ministerio de Sanidad de Gran Bretaña. En 1932 se tuvieron en cuenta los factores sociales: el Comité reconoció la importancia de unos salarios más elevados, de un horario de trabajo más reducido, de la mejora de la dieta y de la mejora de las condiciones de vida a la hora de luchar contra la tuberculosis ${ }^{29}$.

La Gran Depresión obligó al Organismo de Salud de la Sociedad de Naciones a desafiar las opiniones que defendían un papel minimialista de esta institución en relación con la normalización biológica y con la elaboración de la información estadística y de los informes de inspección de las fábricas de los países miembros $^{30}$. Los organismos técnicos y económicos de la Sociedad de Naciones aplicaron unas políticas dirigidas a la mejora de unas condiciones de vida cada vez más deterioradas. La cooperación entre la Organización Internacional del Trabajo y el Organismo de Salud de la Sociedad de Naciones marcó una nueva etapa más innovadora a la hora de desarrollar una medicina social sobre una base económica. Se realizaron una serie de estudios y de conferencias dedicadas a estudiar cómo la salud se veía configurada por la dieta y por las condiciones económicas y de vivienda. El Organismo de Salud juntó sus fuerzas con la OIT para organizar estudios sobre higiene rural y para analizar las relaciones entre la sanidad pública y los seguros de enfermedad.

Marcelino Pascua ${ }^{31}$ formó parte de la secretaría del Organismo de Salud de la Sociedad de Naciones durante el período 1928-30. Estos años fueron cruciales a la hora de formular la política de orientación social del Organismo de Salud. El gobierno español jugó un papel activo al apoyar esta colaboración. En 1930, España propuso la celebración de una Conferencia Europea sobre Higiene Rural. Una importante delegación española asistió a la Conferencia sobre Higiene Rural celebrada en 1931. Su presidente, Gustavo Pittaluga, 
comentó: Esta Conferencia se celebra como consecuencia de los esfuerzos emprendidos por España para remodelar su vida interna. Las recomendaciones incluían programas de seguros y centros rurales de salud ${ }^{31}$. Por lo tanto, este hecho supuso una mayor internacionalización del modelo de Stampar.

La nutrición era un importante objetivo común. El Instituto Internacional de Agricultura (fundado en Roma en 1905) también participaba en estas actividades. En septiembre de 1932, el cambio de política del Organismo de Salud de la Sociedad de Naciones se reflejó en el Informe sobre Depresión Económica y Sanidad Pública. Se subrayaron los defectos de las estadísticas nacionales de carácter agregado a la hora de ocultar la pobreza, y el informe resaltó la necesidad de realizar estudios sobre la morbilidad, la nutrición, los efectos psicológicos del desempleo y los efectos de la pobreza en los jóvenes y los niños. Se crearon comités mixtos compuestos por miembros de diversos organismos de la Sociedad de Naciones, dedicados a correlacionar las informaciones médicas y socio-económicas. Entre los trabajos más innovadores se efectuaron en relación con la mala nutrición de madres, niños y adolescentes. Los defensores de la nutrición, como John Boyd Orr argumentaban que el círculo vicioso de la crisis agrícola y de la mala nutrición urbana se podía solventar aumentando la producción de alimentos saludables, ricos en minerales y en vitaminas. Se trataba de una alternativa científica al escenario tradicional y caritativo de los comedores benéficos. A principios de los años 30 , Rajchman patrocinó programas referidos a una amplia gama de factores sociales que ejercen una influencia sobre la salud, tales como la dieta, las condiciones laborales, el desempleo y la vivienda. ${ }^{32}$

Los expertos en medicina social reconocían que los datos estadísticos en materia de morbilidad y de mortalidad proporcionaban una idea poco adecuada de las condiciones sanitarias. Siguiendo los pasos del Milbank Memorial Fund, los responsables de la Sociedad de Naciones diseñaron un nuevo concepto de «indicadores sanitarios», basados en factores demográficos, médicos, culturales y medioam- bientales, centrando la atención en los factores existentes en localizaciones geográficas restringidas como la densidad de población, la morbilidad, los problemas mentales y el alcoholismo, así como en el gasto y en la disponibilidad de servicios sanitarios. El primero de dichos estudios se realizó en New Haven, Conneticut, en 1936; el segundo se llevó a cabo en el distrito rural de Mezökövesd en Hungría. René Sand inició un ambicioso estudio en Bruselas ${ }^{33}$. Tras abandonar su lugar en el Comité de Salud en 1936, Pittaluga estuvo en la Secretaría del Organismo de Salud de la Sociedad de Naciones en 1937 como uno de los expertos médicos en matcria de refugiados que trabajaba en dicho organismo como consecuencia de los trastornos políticos ocurridos en la década de 1930. El Organismo de Salud estaba en camino de desarrollar un nuevo concepto de «salud positiva», justo en el momento en el que Europa estaba a punto de lanzarse a una guerra total.

\section{LA RUPTURA}

La financiación de la Fundación Rockefeller resultaba crucial para los innovadores programas de Rajchman y servía para financiar en 1933 a un organismo de gran magnitud: el Organismo de Salud de la Sociedad de Naciones contaba con un director medico, 17 directivos técnicos, 35 empleados subordinados, y colaboraba con aproximadamente 100 expertos asociados. La FR financiaba 15 de los 42 cargos técnicos, así como las delegaciones existentes en Polonia, Rusia, Checoslovaquia, Grecia, Hungría e Italia. La Fundación Rockefeller también estaba investigando un programa para establecer un laboratorio permanente dedicado a elaborar unos modelos internacionales ${ }^{34}$. Los fondos de la FR posibilitaban que expertos extranjeros fueran a trabajar a Ginebra y que se contratara a un número superior de empleados británicos y estadounidenses a la cuota establecida por la Sociedad de Naciones.

No obstante, existían críticos feroccs. Buchanan se opuso a los «comités mixtos» y denunció las pretensiones del Organismo de Salud de la Sociedad de Naciones de «constituirse en una autoridad supra-sanitaria respon- 
sable de la supervisión y de la crítica de las administraciones de sanidad pública de todo el mundo ${ }^{35}$. El representante de Estados Unidos ante el Comité de Salud, el Cirujano Jefe Cumming, también mantuvo una postura críti$\mathrm{ca}^{36}$. A esto se añadía la hostilidad del Secretario General Avenol. Al final, Rajchman cortó sus relaciones con la Sociedad de Naciones el 31 de enero de 1939.

Asimismo existía una corriente interna de críticos entre los expertos. En 1925, Stampar recibió a 16 médicos de intercambio que estudiaban la situación en Serbia, Eslovenia y Macedonia, su veredicto fue que las conclusiones del estudio eran estúpidas, de baja calidad y que dichos estudiantes habían convertido su tarea en unas vacaciones ${ }^{37}$. El programa de intercambio llegó a niveles de absurdo cuando el médico y escritor en ciernes, Louis Destouches (más conocido como Céline) autorizó un viaje a unos médicos sudamericanos, destinado a estudiar los modelos de clínicas, los sistemas de alcantarillado, los mataderos y los crematorios, y que se convirtió en una exploración de los placeres más sensuales ofrecidos por las ciudades europeas. Destouches había trabajado previamente para la Misión de la Fundación Rockefeller en Francia y había logrado el apoyo de Gunn. Su sátira cruel de la burocracia de Rajchman, a la que denominaba L'Église, cuya religión era el acercamiento entre los pueblos, y de la Fundación Rockefeller, representada por la Fundación Barell y por el Doctor Darling, resultaba ofensiva, aunque incluso Rajchman se lo tomara con buen humor. La impetuosa diatriba de Céline contra el internacionalismo era un anticipo de los ataques racistas y de la extrema derecha contra las organizaciones internacionales ${ }^{38}$.

La fuente más importante de tensión fue el deterioro de las relaciones entre el radical y expansionista Rajchman, y la Sociedad de Naciones, que se encontraba en fase de desmoronamiento como institución política. En estos momentos, la Fundación Rockefeller aceptó, quizás con demasiada facilidad, la autoridad de la Sociedad de Naciones así como todos los defectos del sistema estatal establecido con motivo del Tratado de Versalles y que estaba condenado a desaparecer. Russell comentó en
1928: La mayoría de los estudiantes de los gobiernos considerarán, en mi opinión, que el organismo de salud de ningún modo debe ser independiente de la Sociedad de Naciones ${ }^{39}$. En 1931, las becas de la Fundación Rockefeller al Organismo de Salud se hubieran detenido de no ser por la intensa actividad de mediación realizada por Rajchman y por su directivo estadounidense Frank Boudreau, que sirvió para proporcionar estabilidad a la institución internacional. Ellos defendían que el cese de la financiación dentro del marco de la depresión suponía una grave amenaza para la propia existencia del Organismo de Salud de la Sociedad de Naciones. Por último, se estableció una nueva división de competencias: la Fundación Rockefeller se concentraría en la investigación y la Sociedad de Naciones en «los problemas de interés y de aplicación inmediata $^{40} . \gg$

En 1934, la opinión del director de la Fundación Rockefeller, Max Mason, era que el Organismo de Salud de la Sociedad de Naciones había tenido «un completo éxito», pero que «el período de ensayo o de demostración ya había finalizado ${ }^{41}$.» Entre 1930 y 1934, se asignaron 700.000 dólares adicionales, debatiéndose en profundidad un «acuerdo de financiación»; y en 1934, Rajchman obtuvo 400.000 dólares USA a repartir en cuatro años y que estaban libres de condiciones en cuanto a su distribución entre las diversas actividades del Organismo de Salud de la Sociedad de Naciones $^{42}$. Este hecho implica que la Fundación Rockefeller contribuyó en parte al desarrollo de los programas radicalmente innovadores de Rajchman, y lo que es más importante, le concedió una autonomía esencial a pesar de todas las reticencias oficiales.

El dilema de Rajchman y del Consejo Internacional de Salud era que compartían la perspectiva de que el progreso de la sanidad pública dependía de los sistemas estatales, a pesar del hecho de que los políticos y los diplomáticos que representaban a los estadosnación eran a menudo indiferentes o abiertamente hostiles ante las cuestiones de sanidad pública. Los grupos de expertos internacionales continuaron siendo vulnerables en una época de auge del nacionalismo: dichos grupos 
debían operar en unas condiciones muy adversas en las que la falta de apoyo gubernamental dificultaba la garantía de imparcialidad. Cualquier futuro organismo internacional debería proporcionar mayor asistencia a los comités de expertos ${ }^{43}$. No obstante, el peligro residía en que el mundo de la investigación podría no tener en cuenta la importancia práctica de las condiciones locales.

En relación con dichas estrategias basadas en el trabajo de los expertos existían problemas en cuanto a la estructura elitista del Organismo de Salud de la Sociedad de Naciones. Aunque la Fundación Rockefeller, tanto en sus unidades de demostración sanitaria como en sus campañas contra la anquilostomiasis, mostraba una valoración del potencial de unas perspectivas más populistas, la asistencia a la sanidad internacional a través de las autoridades estatales y de los expertos en salud pública era elitista. $\mathrm{Ni}$ la Fundación Rockefeller, a pesar de su carácter no gubernamental, ni el Organismo de Salud de la Sociedad de Naciones, apreciaban de forma adecuada la flexibilidad de la organización no gubernamental en el ámbito sanitario en su calidad de proveedores de atención y de «fundamento» de las reformas sanitarias. Ninguna de estas organizaciones abordó las cuestiones de la salud de las mujeres ni de la salud de la familia y, mientras que la Fundación Rockefeller trasladaba prudentemente su centro de interés hacia un programa de control de la natalidad, el silencio del Organismo de Salud en este ámbito resulta difícil de explicar.

Debido a los grandes obstáculos que representaban los intereses de la burocracia y de los estados, los logros del Organismo de Salud resultan impresionantes. El Organismo de Salud de la Sociedad de Naciones fue eficaz a la hora de promover los contactos entre los expertos sanitarios, con inclusión de los expertos españoles, con el fin de desarrollar unas estadísticas internacionales en materia de salud y el estudio de los sistemas de sanidad pública. Sin la financiación otorgada por la Fundación Rockefeller, $y$ sin el entusiasmo personal de sus responsables, como Rose y Gunn, todo esto nunca hubiera ocurrido. Pero la constitución del Organismo de Salud de la Sociedad de Naciones, basada en los estados, obstaculizó la perspectiva de los expertos más dinámicos, y la Fundación Rockefeller se vio dividida entre los criterios de provisión de asistencia y de no injerencia en los principios constitutivos de dicho organismo.

\section{BIBLIOGRAFÍA}

1. L. Murard, P. Zylberman, L'hygiène dans la République. París: Fayard; 1996.p. 554-61.

2. C. Kimmich, German and the League of Nations. Chicago: 1976.p. 80-91.

3. Rockefeller Archive Center (= RAC), RF 1.1 $1100 / 22 / 184$. Rose a William Fredcrick Russell, 13.VIII.22, citado por Strode, Memorandum on Health Organization. 6.VIII. 28.

4. RAC RF RF $1.1 / 100 / 22 / 184$. Rose a Russell, 13.VIII.22, citado por Strode, 6.VIII.28.

5. RF 1.I / 712/3/16, informe de Rose y Gunn, «Public Health Situation in Czechoslovakia. 1919.

6. M. A. Balinaka. Une vie pour l'humanité. Ludwik Rajchman, 1881-1965. París: 1995.

7. E. Fee. Designing Schools for Health in the United States. En: A History of Education in Public Health». E. Fee y R. Acheson eds. Oxford; 1991.p. 155-94;

8. Fee, «Disease and Discovery. A History of the Johns Hopkins School of Hygiene and Public Hcalth 1916-1939. Baltimore; 1987.

9. RAC RF $1.1 / 100 / 20 / 175$, Ilowell a Russell, 27.VI.30.

10. Sociedad de Naciones, Health Committee. Minutes of the Third Session Held at Geneva form Monday, September 29 th to Saturday, October 4th, 1924, Ginebra; 1924.

11. J. M. Piñero et al. (eds.), Diccionario histórico de la ciencia moderna en España, Barcelona;

12. Bibliography of the Technical Work of the Health Organisation of the League of Nations, 1920-1945. Bull IJealth Organisation 1945; 11: 1-235.

13. RAC FR 1.1/7.2/2/13. Rose a Gunn, 3.I.22.

14. RAC FR $1.1 / 100 / 20 / 165$. Rose a Rajchman.

15. RAC FR 1.1/100/20/164, compromiso del $1 / 3 / 29$ que sirvió para asignar 350.700 dólares USA a la elaboración de estadísticas médi- 
cas y aproximadamente 750.000 dólares USA para el programa de intercambios. Se comprometieron casi 750.000 dólares USA durante el período 1930-34.

16. Cf. Balinska, Rajchman.p. 119-20.

17. RAC RF $1.1 / 7.2 / 2 / 12$, carta de Rajchman a Gunn de 9.XII.21 1.1 / 100/20/165, Rose a Gun en relación con la contratación de Gunn.

18. RF $6.1 / 1.1 / 46 / 524$, Slobodan Lang, A Corner of History. Andrija Stampar. 18881958 «Un hito en la historia. Andrija Stampar. 1888-1958»), Prev Med, 1975; 4: 591-5.

19. M. Grmek ed. «Selected Papers of Andrija Stampar» Zagreb; 1966.

20. N. S. Beeson (ed.), Henry E. Sigerist: Autobiographical Writings. Montreal; 1996.p. 31-3.

21. RF 1.1/710/1/5 «Public Health in Zagreb 1925. Se asignaron 145.000 dólares USA en 1925 para el programa de Zagreb.

22. C. Prauenitz. The Teaching of Preventive Medicine in Europe. Oxford; 1933.p. 141-8.

23. Ibid. Russell a Strode, 13.VI.28.

24. RAC RF 1.1/100/20/172. Russell, 5.III.31.

25. Ibid. SMG Diary (»Diario SMG»), 2.VIII.28.

26. RAC RF 1.1/100/20/177, Concesión de una subvención para la formación en materia de estadísticas vitales y de salud pública.

27. J. Orensanz. España. Anuario Internacional de Salud 1927;. 3.p. 619-48.

28. I. Löwy. Testing for a Sexually Transmissable Disease 1907-1970: the History of the Wassermann Reaction» V. Berridge y P. Strong (eds.),

29. AIDS and Contemporary History. Cambridge; 1993.p. 74-92.

30. Weindling, The Politics of International Coordination. AIDS: 93-107.

31. Zinsser Papers. carta de Zinsser a Rajchman, 25.I.37, 20.X.37, 13.IV.37, 22.I.38. Zinsser a Plotz, 22.IX.37, 22.XII.37.

32. Zinsser, As I Remember Him.p. 368-9.

33. Zinsser a Giroud, 21.IX.37 en relación con los lotes de vacunas para Sergent en Argel.

34. «Zinsser Papers. Zinsser a Plotz, 21.XI.38.

35. Report of the Inter-governmental Conference on Biological Standarization. Quarterly Bull Health Organisation League of Nations 1935; 4.
36. Archivos de la Sociedad de Naciones, Ginebra, R 6078-9 en relación con la tipificación de las vitaminas.

37. E. Burnet, General Principles Governing the Prevention of Tuberculosis Bull Health Organisation 1932; 1:489-663.

38. La Fundación Rockefeller rechazó una solicitud para financiar la nueva enciclopedia en 1924, ver los archivos OIT de Ginebra (en adelante, denominados los archivos OIT). Relaciones HY 104 con la Fundación Rockefeller. Para las propuestas en materia de ciencias sociales, ver RAC RF 6.1 / 1.1 / 38 / 465, S. H., Walker memo Aug-Sept 1935.

39. J. Bernabeu Mestre, «Marcelino Pascua desde la perspectiva histórica».

40. G. Clavero González, «La labor de Marcelino Pascua en su entorno», «Las estadísticas demográfico-sanitarias, I Encuentro Marcelino Pascua», Madrid, 1992;

41. E. Rodríguez Diana y J. Bernabeu Mestre, «El legítimo criterio aritmético de los métodos cuantitativos en la sanidad pública»;

42. F. Sánchez, Granada, 1996.

43. Sociedad de Naciones. European Conference on Rural Hygiene. Ginebra; 1931, vol. 2.

44. Documentos de la Sociedad de Naciones, Publicaciones de Investigación, carrete 3:9, «Trabajos del Comité de Salud en su $19^{\mathrm{a}}$ Sesión, Ginebra, 10-15 de octubre de 1932. OIT HY 200, HY $200 / 2 / 2$. Colaboración del OIT y de la Sociedad de Naciones. P. J. Weindling, «Social Medicine at the League of Nations and International Labour Office compared» («Estudio comparativo de la medicina social aplicada por la Sociedad de Naciones y por la Organización Internacional del Trabajo"), Weindling. (ed.), International Health Organisations.p.134-153. Weindling, «The Role of International Organisations in Setting Nutritional Standards in the 1920's and 30's» («El papel de los organismos internacionales a la hora de establecer unos modelos de nutrición en las décadas de 1920 y 1930 »); A. Kamming y A. Cunningham (eds.), The Science and Culture of Nutrition. Amsterdam; 1995.p. 319-32.

45. K. Stouman y I. S. Falk. Indicadores sanitarios. Un estudio de los indicadores objetivos de la salud en relación con el medio ambiente y la higiene. Quarterly Bull Health Organisation 1936;5: 901-96. 
46. Stouman, «Health Indices Established in an Experimental Study of the City of Brussels. «Bull Health Organisation 1938; 7: 122-67.

47. RAC RF $1.1 / 100 / 20 / 169$. Carta de Gun a Russell, 16.VII.25.

48. G. S. Buchanan, International Cooperation in Public Health. Its Achievements and Prospecis. Londres; 1934. p. 29.

49. Balinska, Rajchman, p. 125.

50. RAC RF 1.1/100/20/169. Carta de Gunn a Russell, 16.VII.25.
51. Balinska, Rajchman, p. 176-9.

52. RAC RF $1.1 / 100 / 21 / 173$. Carta de Russell a Strode, 13.VI.28.

53. RAC RF 6.1/1.1/37/467. Diario de G. K. Strode, 13.V.37, reunión con Rajchman.

54. RAC RF 6.1/1.1/37/463. Carta de Mason a Avenol, 31.XII.34.

55. RAC RF 6.1/1.1/37/464. Auditorías del período 1934-37.

56. Cf. Opiniones de Madsen, RAC RF 6.1/1.1/ 37 / 469, Madsen, 30.VI.34. 